\title{
Simulation and real data analysis of the LHAASO-WCDA dynamic range extension system
}

\author{
Xiurong Li*; Cheng Liu, Zongkang Zeng, Xiaochuan Chang, Min Zha, Zhiguo Yao, \\ Hangrong Wu, Huihai He, Zhen Cao, Hongbo. Hu, for the LHAASO collaboration \\ Key Laboratory of Particle Astrophysics, Institute of High Energy Physics, Chinese Academy of \\ Science, Beijing 100049, China. \\ E-mail: lixreihep.ac.cn
}

\begin{abstract}
The Water Cherenkov Detector Array (WCDA) is one of the major components of the Large High Altitude Air Shower Observatory (LHAASO). The installation of the first pool of WCDA is already finished and normally operated. Except for the initially designed 8-inch PMTs (big PMTs) of WCDA installed, each of the 900 cells of the first pool was installed another 1.5-inch PMT (small PMTs) to extend the dynamic range, which is called the WCDA dynamic range extension system (WCDA++). The 900 1.5-inch small PMTs work together with WCDA big PMTs and WFCTA and KM2A to measure the energy spectra of cosmic rays with energy from $\mathrm{TeVs}$ to $10 \mathrm{PeV}$. This paper presents the data analysis and simulation of the system, such as the single rate study, the match of single-channel triggered hits of big and small PMTs, the match of cosmic ray events, the core reconstruction, and full Geant 4 simulation and fast simulation based on Geant4.
\end{abstract}

36th International Cosmic Ray Conference -ICRC2019-

July 24th - August 1st, 2019

Madison, WI, U.S.A.

\footnotetext{
* Speaker.

${ }^{\dagger}$ This work is supported by the National Key R\&D Program of China (No. 2018YFA0404201), (No. 2018YFA0404202), the Chinese Academy of Sciences, the Key Laboratory of Particle Astrophysics, IHEP, CAS.
} 


\section{Introduction}

The Large High Altitude Air Shower Observatory (LHAASO) [1] is a large hybrid extensive air shower(EAS) array being constructed at Haizi Mountain, Daocheng, Sichuan province, China. It is composed of three subarrays, i.e., $1.3 \mathrm{~km}^{2}$ array (KM2A), 78,000 $\mathrm{m}^{2}$ water Cherenkov detector array (WCDA) and 12 wide-field air Cherenkov/fluorescence telescopes (WFCTA). LHAASO is designed to search for cosmic ray sources by the measurement of $\gamma$ rays and to study cosmic ray physics from $\mathrm{TeVs}$ to a few hundreds of PeV [2]. LHAASO will accurately measure the energy spectra of cosmic rays for single elements and improve the chaotic situation in measurement of energy spectra, with three major detector arrays: LHAASO-WCDA, LHAASO-KM2A, LHAASO-WFCTA.

The WCDA is 3,120 detector units divided into 3 separate water pond arrays with $4.4 \mathrm{~m}$ depth [3]. Two of them with an effective area of $150 \times 150 \mathrm{~m}^{2}$ contain 900 detector units each. The third pond with an area of $300 \times 110 \mathrm{~m}^{2}$ contains 1,320 detector units. Each detector unit has a $5 \times 5 \mathrm{~m}^{2}$ area divided by black plastic curtains $(3.3 \mathrm{~m} \times 4.7 \mathrm{~m})$ vertically hung in the water. The No.1 pool has 900 units, equipped by a large 8 -inch PMT for timing and a small 1.5-inch PMT. The 900 units of small 1.5-inch PMTs were installed to extend the dynamic range of the detector, which is called the WCDA dynamic range extension system (WCDA++). The left of Fig.1 is the sketch map of two cells of the system including big and small PMTs, and the right shows the geometry of a small PMT including the water proof system. The small PMTs of 1st WCDA pool will work together with WCDA big PMTs and WFCTA and KM2A and to measure the energy spectra of cosmic rays from $\mathrm{TeVs}$ to $10 \mathrm{PeV}$, which can give good energy scaler between spacebored experiments and ground-based measurements. The system detects the Cherenkov light which produced in water by cascade process of secondary particles that falling into the water cell. The number of photon electrons (NPE) of PMTs reflects the density and energy of secondary particles, which is able to reconstruct the core position and is sensitive to the identity of primary particles. The directions of low energy cosmic ray can be reconstructed with time and charge information of big PMTs, while the core position and particle identification and energy reconstructed can be improved to use big and small PMTs together for the events with big PMTs saturated.

In February, 2019, the detector installation of No.1 WCDA pool was completed then purified water were injected to the water pool which reaches $4.4 \mathrm{~m}$ after more than. The dry run started on February and the normal operation of the first pool with water started in the end of April. The data of single-channel triggered hits has been taken for small PMTs, while the data of both of cosmic ray events and single-channel triggered hits has been taken for the big PMTs. Both data analysis and simulation has been done to understand the detectors and also cosmic rays detected.

\section{Data analysis}

Currently, the data taking mode for the small PMTs of 1st WCDA pool is single-channel trigger mode. A single-channel trigger is generated if the anode signal amplitude of a small PMT exceeds the value corresponding to about 20 number of photon electrons. Normally the data taking for the big PMTs is cosmic ray event triggered, generated if there are tens of big PMT hits in hundreds of ns. The data taking of big PMTs temporally changes to the single-channel trigger 

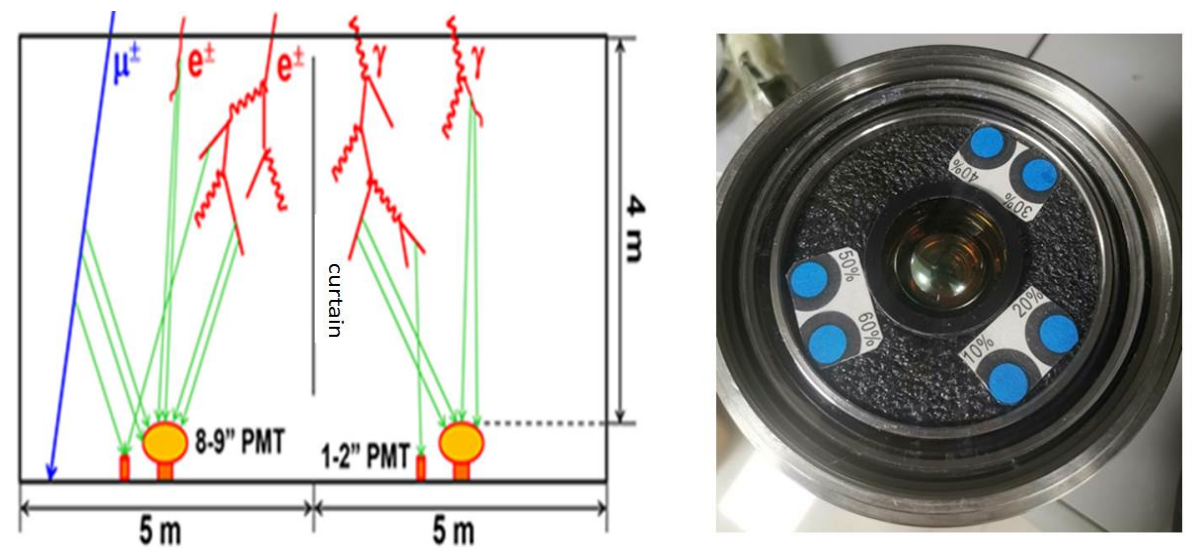

Figure 1: The left is sketch map of two cells of 1st WCDA pool; The right is the geometry of the small PMT including the water proof system.

mode if there are Gamma burst alarms, with the threshold for each big PMT about 1/3 of single photon electron. Both the single-channel trigged hits and cosmic ray events data were analyzed and matched with big and small PMTs, the core of cosmic rays were reconstructed using big and small PMTs together.

\subsection{Trigger rate of small PMTs}

The small PMTs work at the gain about $2 \times 10^{5}$, the threshold for the single-channel trigger is about 20 NPE. The left of Fig. 2 shows the trigger rate at different time of one small PMT when it was merged by water. It can be seen the event rate increased suddenly when it was merged by water then increase gradually when the water depth is not too high. The right of Fig. 2 shows the triggers rate of different PMTs about $3 \mathrm{~Hz}$ after the normal operation with $4.0 \mathrm{~m}$ water upper the top surface of big PMTs.
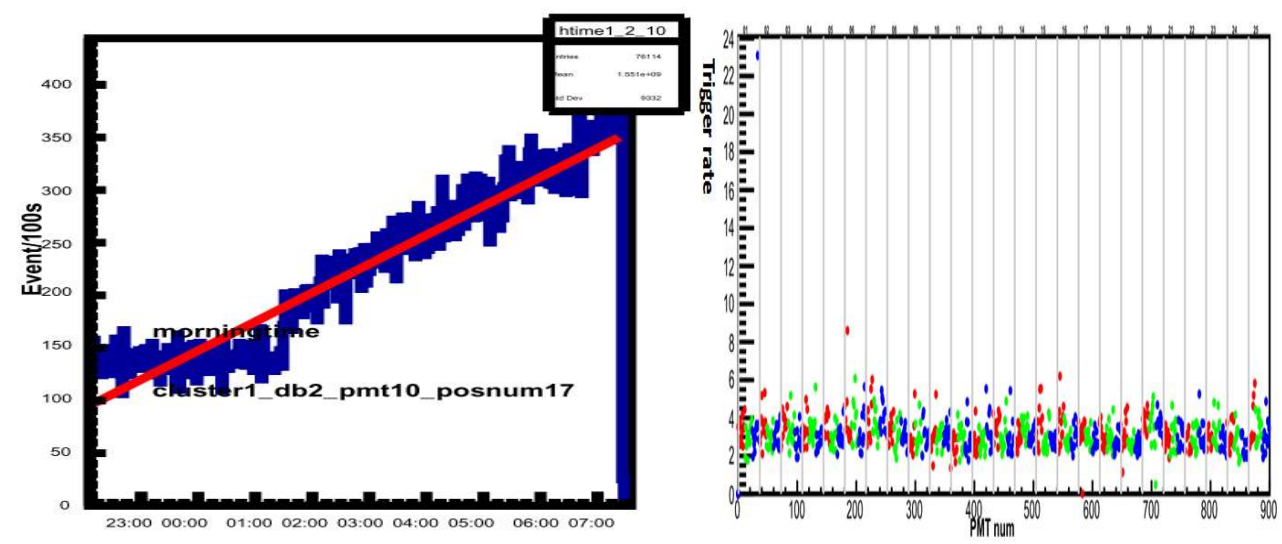

Figure 2: The left is the event rate at different time of one small PMT when it was merged by water; The right is the triggers rate of different PMTs after the normal operation with $4.0 \mathrm{~m}$ effective water depth.

\subsection{Match of single-channel triggered hits}

The single-triggered hits of big and small PMTs were separately taken. Most of the hits of 
small PMTs are caused by random muons as the threshold is bigger than 20 NPE. The hits of big and small PMTs in the same cell with little time difference can be the signal caused by the same particle hitting around the small PMT, which can used to do calibration and study the performances of detectors. We selected the single-triggered hits of big and small PMTs in each cell in a narrow time window, and found matched hits with the time difference about the time resolution. The left of Fig. 3 is the dynode amplitude of a big PMT from the matched hits, and the right is anode amplitude of matched small PMT hits. The dynode amplitude of the matched big PMTs hits has a narrow shape which is much clear than the peaks in the random triggered hits. There is also a hump on the anode amplitude distribution of the matched small PMTs hits. More work will do to use these information to do absolute calibration and get the difference of different cells.
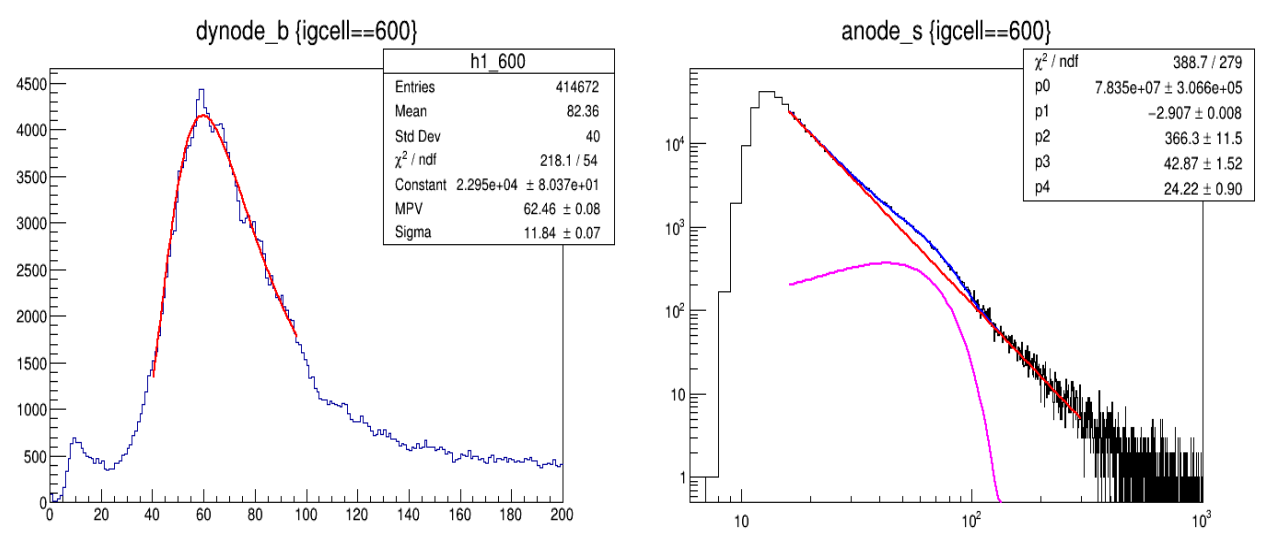

Figure 3: The left is the dynode amplitude of a big PMT from the matched hits; The right is anode amplitude of the matched small PMT hits.

\subsection{Match of cosmic ray events with big and small PMTs}

The cosmic ray events of big and small PMTs will be taken together later. Temporally the data of cosmic ray event is taken with big PMTs, and the small PMTs take data of single-channel triggered. We matched the cosmic ray events with big and small PMTs, by selecting the hits of small PMTs in a time window near to each cosmic ray event time triggered by big PMTs. The selected small PMT hits and the previous big PMT hits of the event are filled together to root file as an event for analysis. The direction and core of the cosmic ray with no big PMT hits saturated are reconstructed using charge and time information of the big PMT hits. The hits of big PMTs saturate with NPEs about more than 6000. For the cosmic ray event with high energy which cause big PMTs saturated, the saturated big PMTs hits are replaced by relative small PMTs for reconstruction and analysis.

Fig. 4 is the event display of a cosmic ray event detected. The left top of Fig. 4 is the time distribution of the big PMT hits. The color of middle top of Fig. 4 represents the time of different big PMT hits at different position, and the size represents NPE of the big PMT hits, with diamonds represents the saturated big PMTs hits. The right top of Fig. 4 is the NPE of big PMTs and left bottom is NPE of small PMTs. The middle bottom of Fig. 4 shows the correlation of NPE of big and small PMTs, and middle bottom of Fig. 4 shows the NPE of this event with NPE of small 
PMTs replacing saturated NPE of big PMT. The direction and core position of the cosmic event is reconstructed and showed on middle bottom of Fig. 4.
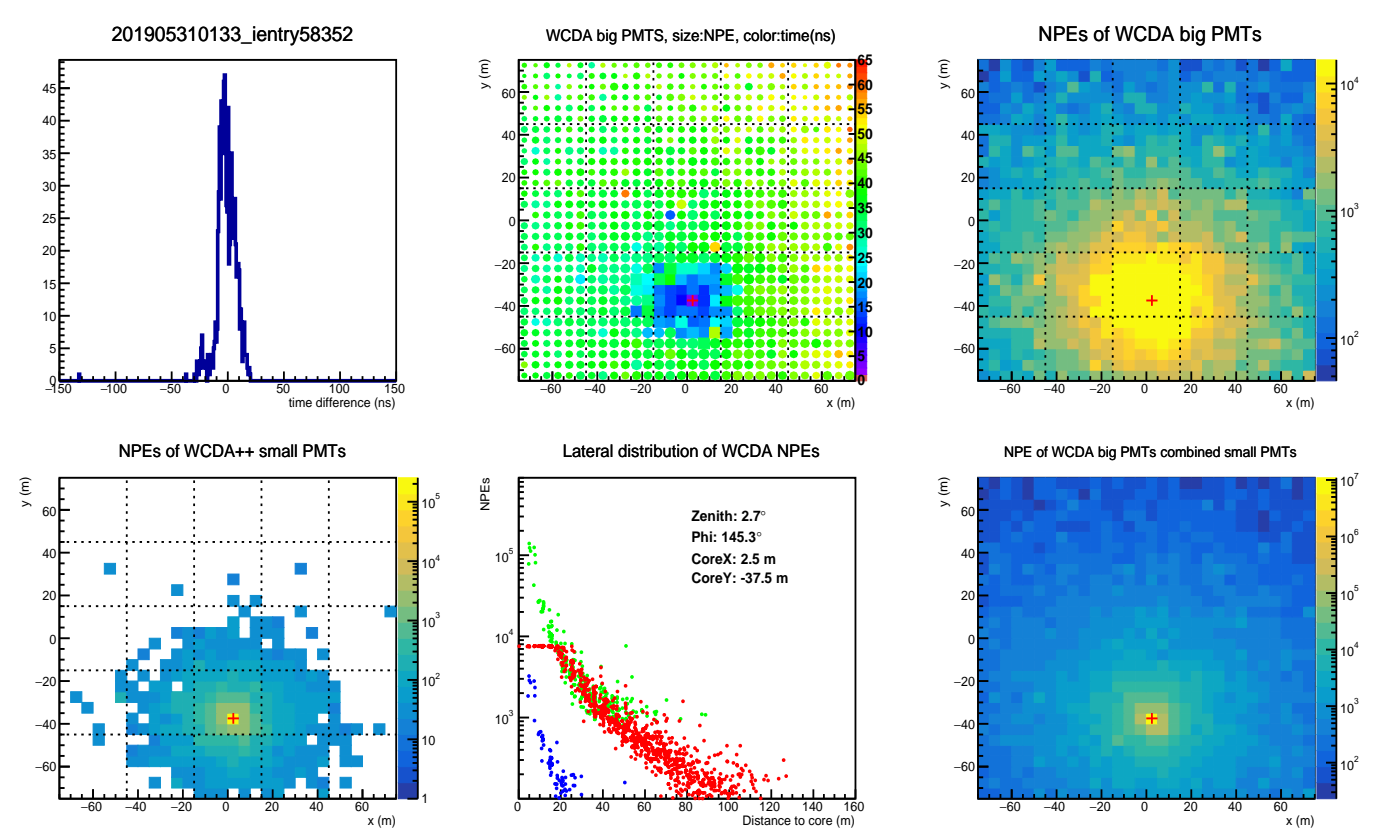

Figure 4: Event display of a cosmic ray event detected with big and small PMTs

\section{Simulation}

The full simulation of cosmic rays are divided into two part. First Corsika simulation [4] are used to get secondary particles, then the secondary particles are simulated with Geant 4 to get performance of the WCDA++ detectors including big and small PMTs. Here the Geant4 simulation [5] will be described, including the detector setup, the simulation of muons and cosmic rays with different energy, and fast simulation based on Geant 4 .

\subsection{Detector set up of the small PMTs}

The setup of big PMTs and the 1st pool of WCDA and also the physics process are same with the standard code of WCDA simulation with big PMTs, where the WCDA++ simulation code inherited from [6]. We try to make the set up of the small PMTs in the simulation same as the real detectors. For each unit, the small PMT is set at west-north of the big PMT with $22 \mathrm{~cm}$ distance. The small PMTs are made water prof with stainless steel and quartz glass on the face of PMT. There is a dark circle on each small PMT face to make the diameter of the photocathode about $3.2 \mathrm{~cm}$ open for photon collection. There is air gap between the water proof quartz glass and the glass face of small PMT. Thus there is total reflection effect that photons with angle bigger than 48 degree can not go through to the PMT. The size and height of different layers on the small PMTs for simulation are made same with the real experiment set up showed on right of Fig. 1. The shape of the PMT glass is set same with that given by the company. The PMT quantum efficiency was 
supplied by measurement with PMT glass on the photocathode. The PMT glass is set with the real refractive index of glass but with no absorption. The optical model between water and glass and air for the small PMTs are automatically set by Geatn4 as the surfaces are smooth. The optical performance such as the total reflection effect were carefully checked to make sure it right. Photons that arrive the photocathode are converted into Npe with the probability as the PMT efficiency.

\subsection{Simulation of muon backgrounds}

Some random muon backgrounds were simulated to compare with the matched single-channel triggered signal of big and small PMTs. The angle distribution and energy spectrum of the muons were got from secondary muons far from the core generated form corsika simulation of cosmic rays [7]. The muon backgrounds were randomly set in the positions with the horizontal distance to the small PMT less than $10 \mathrm{~cm}$ were simulated. The left of Fig. 5 is NPE distribution of the big PMT with requiring NPE of the small PMT bigger than 20. The right of Fig. 5 is NPE of the small PMT with requiring Npe bigger than 20. The shapes of the simulation signals are similar with the real data, and more work about simulation and calibration will be done to get same NPEs of simulation and real data.
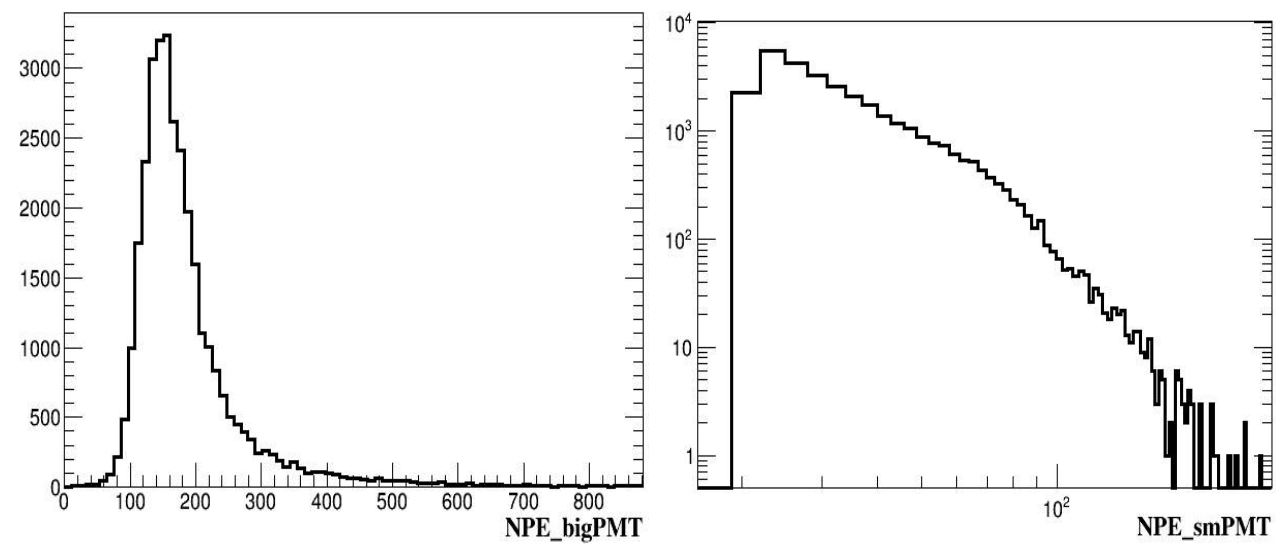

Figure 5: The left is the NPE of the big PMT caused by the random muon background simulation; The right is the NPE of the small PMT caused by the random muon background simulation.

\subsection{Simulation of cosmic events}

Some comic ray Protons with Energy of 1-500TeV and theta angle less than 13 degree were simulated by CORSIKA and WCDA++ simulation code to study the performance. The cascade processes of primary particles in the atmosphere are simulated by the CORSIKA program with the version of 6990. The EGS4 model is chosen for the electromagnetic interaction. The QGSJET02 and GHEISHA models are chosen for the high and low energy hadronic processes respectively.

The secondary particles generated were simulated by the WCDA++ simulation code to get the NPE of each big and small PMTs and the arriving time of photon at the PMTs. The core position were set at the center of 1st WCDA pool for simulation. Left of Fig. 6 is the ratio of Npe of big PMTs to Npe of small PMTs in the same cell versus the Npe of big PMTs, with the profiled mean value quite consistent. The core reconstruction were done using big and small PMTs together, by 
replacing the NPE of big PMTs with NPE of small PMTs multiplying a factor. The right of Fig. 6 is the core shift between input and reconstruction values with simple weight method. It can be seen the core shift is less than $3 \mathrm{~m}$ for the protons with energy above $100 \mathrm{TeV}$. More reconstruction methods will be applied to improve the core reconstruction error for cosmic rays with low energy.
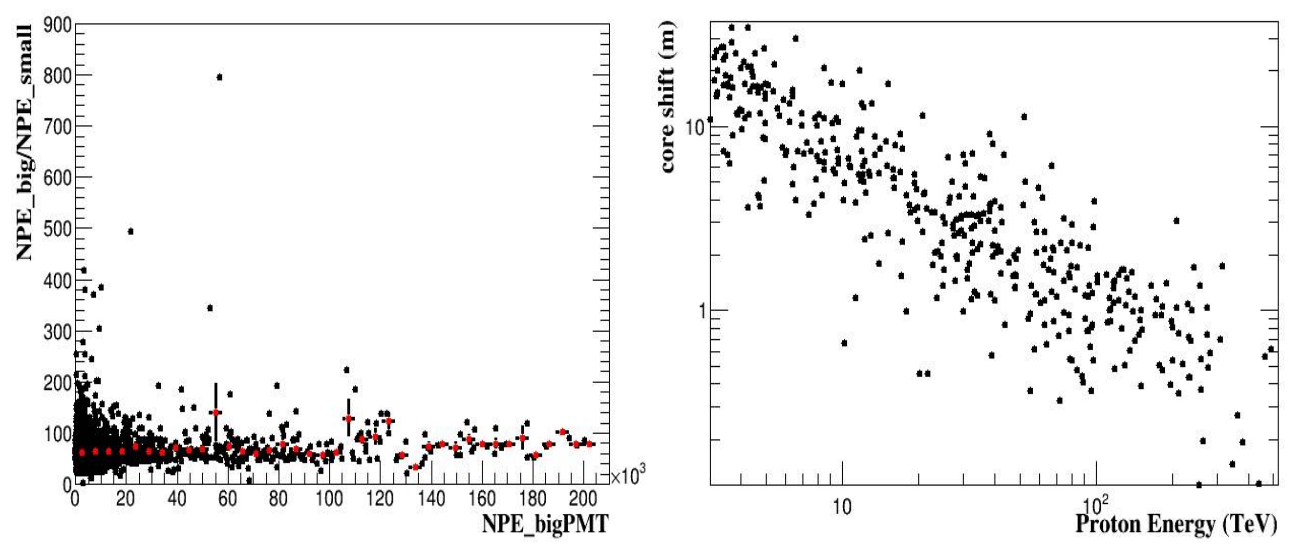

Figure 6: The left the ratio of Npe of big PMTs to Npe of small PMTs in the same cell versus the Npe of big PMTs; The right is the core shift between input and reconstruction values with simple weight method.

\subsection{Fast simulation based on Geant4}

Different methods are used to speed up the Geant 4 simulation to get NPE and time information of PMTs same with full simulation. The quantum efficiency of PMT depends on the wavelength of photons, thus the photons can be randomly killed according to the PMT quantum efficiency before tracking. Another method is estimating whether the Cerenkov photons can hit the PMTs at each step of the charged particle simulation, doing full simulation of the photons at the PMT directions and other with thinning. The two methods can speed up the Geant 4 simulation for more than 50 times, with NPE and PMT arriving time information similar with full simulation. The left of Fig. 7 is the NPE of a big PMT caused by $2 \mathrm{GeV}$ muons, simulated with full method and the two fast methods. The right of Fig. 7 is the NPE of a big PMT caused by $2 \mathrm{GeV}$ gamma, simulated with full and fast methods. The fast simulation can be further developed with speed much faster.

\section{Summary}

The installation of the first pool of WCDA is already finished and normally operated. The data analysis and simulation of WCDA++ has been done. Both the single-channel trigged hits and cosmic ray were analyzed and matched with big and small PMTs, the core of cosmic rays were reconstructed using big and small PMTs together. The Geant4 simulation of muons backgrounds were done and got similar signal shapes with the real data. Cosmic ray events were simulated and the core was reconstructed with big and small PMTs together to get core shift less than $3 \mathrm{~m}$ for Protons with energy bigger than $100 \mathrm{TeV}$. More simulation and analysis will be done to understand the calibration and performances of detectors. More reconstruction methods will be applied to get better results. 

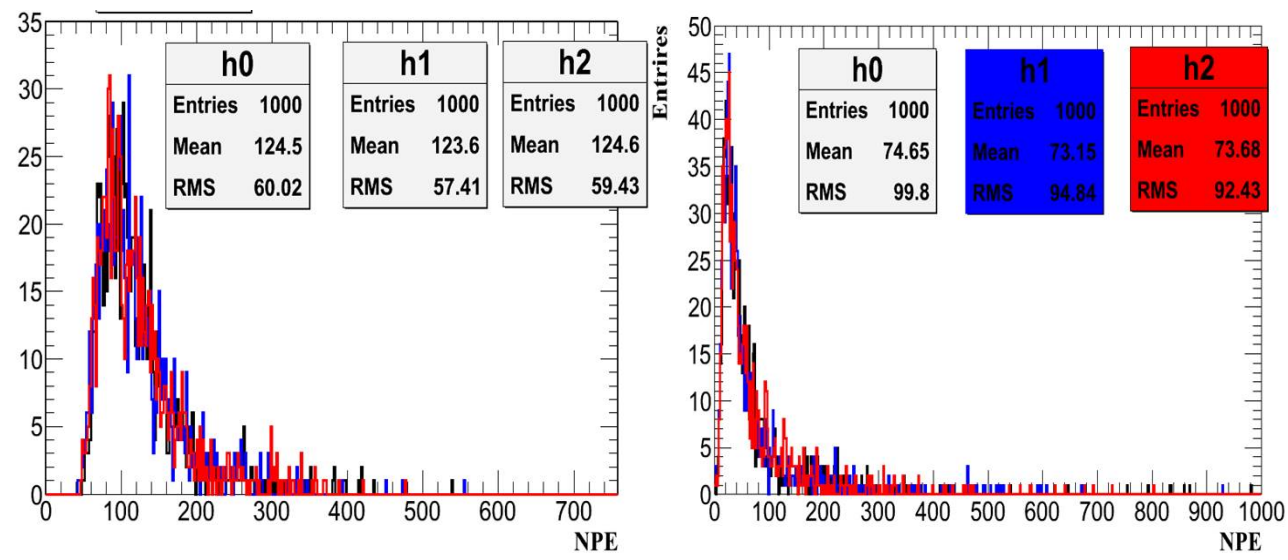

Figure 7: The left is the NPE of a big PMT caused by $2 \mathrm{GeV}$ muons, simulated with full method and the two fast methods. The right is the NPE of a big PMT caused by $2 \mathrm{GeV}$ gamma simulation

\section{References}

[1] He,H.H., for the LHAASO Collaboration, 2018, Radiation Detection Technologyand Methods 2, 7

[2] Z.Cao (for LHAASO Coll.), Chin.Phys.C34 (2010) 249-252.

[3] MJ. Chen, .et al, Status and first result of LHAASO-WCDA, ICRC 2019.

[4] D. Heck and T. Pierog, Extensive Air Shower Simulation with CORSIKA: A Userąŕs Guide, 2015.

[5] S.Agostinelli,etal,Geant4 Collaboration, Nucl.Instrum.Methods, Phys.Res.A,506(2003): 250íC303.

[6] M. Zha, Hc. Li, et al, Comparison of measured and simulated data with LHAASO-WCDA run data, Proceeding of Science, ICRC 2019.

[7] Hui-Cai Li,Zhi-Guo Yao,Chun-Xu Yu, et al. Chinese Physics C, 2017, 41(2): 26002-026002. 